\title{
Community Hand-Dug Wells for Trachoma: A Cluster-Randomized Trial
}

\author{
Solomon Aragie, ${ }^{1} \dagger$ Sintayehu Gebresillasie, ${ }^{1} \dagger$ Ambahun Chernet, ${ }^{1}$ Ayalew Shiferaw, ${ }^{1}$ Zerihun Tadesse, ${ }^{1}$ Mulat Zerihun, ${ }^{1}$ \\ Nicole E. Varnado, ${ }^{2}$ Sun Y. Cotter, ${ }^{2}$ Dionna M. Wittberg, ${ }^{2}$ Zhaoxia Zhou, ${ }^{2}$ Elizabeth Kelly Callahan, ${ }^{3}$ Scott D. Nash, ${ }^{3}$ \\ Kristen Aiemjoy, ${ }^{2,4}$ and Jeremy D. Keenan ${ }^{2,5 *}$ \\ ${ }^{1}$ The Carter Center Ethiopia, Addis Ababa, Ethiopia; ${ }^{2}$ Francis I Proctor Foundation, University of California, San Francisco, San Francisco, \\ California; ${ }^{3}$ The Carter Center, Atlanta, Georgia; ${ }^{4}$ Department of Epidemiology and Biostatistics, University of California, San Francisco, San \\ Francisco, California; ${ }^{5}$ Department of Ophthalmology, University of California, San Francisco, San Francisco, California
}

\begin{abstract}
The WHO recommends improving access to water as part of a comprehensive strategy for elimination of trachoma as a public health problem; however, this recommendation is not based on evidence from randomized trials. In a region of Ethiopia with hyperendemic trachoma, seven communities were randomized to a hand-dug well (HDW) and seven communities to no intervention to determine the impact of HDWs on the community prevalence of ocular chlamydia infection (primary prespecified outcome). All communities continued to receive government hygiene and sanitation services and outreach. Participants were not masked, given the nature of the intervention, but laboratory personnel were masked to treatment allocation. Hand-dug wells were successfully built in six of the seven communities; five of these wells were still functional at the conclusion of the trial. At the end of the trial, an average of $74 \%$ of households reported traveling $<30$ minutes to collect water in the HDW arm, compared with $45 \%$ in the control arm, and the daily volume of water used for hygiene was similar (e.g., mean of $0.7 \mathrm{~L}$ per person in each arm). The pseudo-median prevalence of ocular chlamydia among 0 - to 5 -year old children at the 24 -month visit was $23 \%$ in the HDW group and $13 \%$ in the control group $(P>0.99)$. This small cluster-randomized trial provided no evidence to suggest that simply constructing HDWs, in the absence of other hygiene promotion activities, is effective for reducing transmission of ocular chlamydia.
\end{abstract}

\section{INTRODUCTION}

The WHO recommends improving access to water as one component of a broader strategy for elimination of trachoma as a public health problem. Increasing the number of water points in a community presumably reduces travel times associated with water collection and increases the overall volume of water available to a community, thereby increasing the availability of water for face washing. Washing faces is thought to be important for trachoma elimination because the infectious agent of trachoma, Chlamydia trachomatis, is found in ocular and nasal secretions. ${ }^{1}$ Numerous observational studies have found that individuals with unclean faces, usually defined as ocular discharge, nasal discharge, or flies on the face, are more likely to have trachoma than those with clean faces. ${ }^{2}$ Although the causal directionality of this observation is not well established (i.e., whether an unclean face causes trachoma or whether ocular chlamydia infection causes discharge-and hence, an unclean face), washing children's faces is specifically encouraged because the highest prevalence of trachoma and ocular chlamydia infection is found among children. ${ }^{3,4}$

Although improving access to water has long been a part of the WHO's elimination strategy, few interventional studies have been performed to assess whether building additional community water points is effective for reducing ocular chlamydia or trachoma. ${ }^{5}$ And whereas few would discourage construction of new water points, trachoma programs have limited resources and must decide whether water infrastructure has a favorable cost-benefit ratio for the goal of trachoma control. In this exploratory cluster-randomized trial, we assess whether construction of community water points alone, in the absence of any other hygiene promotion

\footnotetext{
*Address correspondence to Jeremy D. Keenan, 513 Parnassus Ave., Box 0412, University of California, San Francisco San Francisco, CA 94143. E-mail: jeremy.keenan@ucsf.edu
}

†These authors contributed equally to this work. activities, is effective in reducing ocular chlamydia in children. Cluster randomization was used because a community water point is inherently a community-level intervention and because trachoma is an infectious disease with considerable potential for within-village transmission. We hypothesized that construction of a hand-dug well (HDW) would increase the volume of water available for hygiene purposes, leading to an increase in face-washing and reduction in the transmission of ocular chlamydia.

\section{METHODS}

Ethics. Ethical approval was obtained from the University of California, San Francisco, Emory University, the Ethiopian Ministry of Science and Technology, and the Food, Medicine, and Health Care Administration and Control Authority of Ethiopia. Informed consent was obtained from community leaders before randomization and from guardians of children after randomization. Verbal consent was obtained because of the high levels of illiteracy in the study area.

Study design. A parallel-group cluster-randomized trial was conducted from April 2014 to April 2016 in Goncha Siso Enese woreda, Amhara region, Ethiopia. Communities that had been treated with 7 years of community-wide azithromycin distributions as part of the TANA I and TANA II trials were randomized to either a community HDW or to no intervention. ${ }^{6}$ The primary outcome was the prevalence of ocular chlamydia infection, assessed in a random sample of children aged $0-5$ years 2 years after water point construction. The trial was registered with clinicaltrials.gov (\#NCT02373657).

Setting. The study area consisted of agrarian communities. The main crop is Eragrostis tef, an important grain for Ethiopia that grows abundantly in the area. Based on a baseline survey of 388 randomly selected households from the intervention communities, 331 (85\%) reported access to a HDW and 163 $(42 \%)$ to an unprotected spring; $39(10 \%)$ had access to surface water only. Of surveyed households, $259(67 \%)$ reported needing $<30$ minutes to collect water, but only $170(43 \%)$ 
reported having adequate water for hygiene purposes. Water availability was reduced in the study area during a severe drought in 2015-2016.

Participants. Similar to TANA II, the randomization unit was the state team, a government-defined demographic unit consisting of approximately 50 households and termed community in this report. Communities were drawn from three arms of the TANA II trial: annual mass azithromycin distributions, biannual mass azithromycin distributions, and annual azithromycin distributions targeted to preschool children. Communities were eligible if $\leq 1$ functional improved water source (e.g., HDW, borehole, and protected spring) was present within the borders of the community and a suitable area for a HDW could be identified. Remote communities, defined as those that were so far from the main road that a site visit could not be made without staying overnight, were excluded. All willing households in the eligible communities were included in the census for the trial.

Randomization and masking. Communities were randomized in a 1:1 ratio to a community HDW or no intervention, stratified by their randomization arm from the TANA II trial. Randomization was performed in May 2014 by J. D. K. using the rand function in Microsoft Excel (Redmond, WA). Allocation was concealed by enrolling all communities before randomization. Because of the nature of the intervention, study participants were not masked. The field examination teams were not informed about the purpose of the study or randomization allocation. Masking of all laboratory staff was achieved by labeling all specimens with a 6-digit random number.

Intervention. Local water officials recommended that we construct HDWs based on their knowledge of the study area and given the limited budget of the study. A geologic survey was performed with the assistance of local water officials in April 2014 in all eligible communities to identify locations where a HDW would likely provide water. An attempt was made to find locations for water points that were close to dwellings and far from existing water points, but in practice, this was not always possible. A potential site for a HDW was identified in 14 communities, which were subsequently enrolled in the present trial and randomized (Supplemental Figure 1). Local contractors licensed by the local water office were hired to build the HDW according to local specifications. All HDWs were capped, equipped with a hand pump, and protected by a fence. Local community members and the woreda water office members participated in the construction and were responsible for the maintenance of the water points after their construction. All community members could access the water point at any time of day, 7 days per week, regardless of study participation. No water use tariffs were levied. Although no formal assessment was conducted, the trial coordinator received no complaints about poor water taste. Several other hygiene measures had been planned for the intervention communities, but these proved difficult to implement given funding constraints, and thus no other sanitation or hygiene activities were implemented as part of the study. Study communities did not receive mass azithromycin distributions during the follow-up period. No water interventions were performed in the control communities during or after the trial. No changes to the intervention or methods occurred during the study period. Recording of adverse events was performed via a passive system similarly to the TANA II trial in which community members would inform local health extension workers, who would then inform the study coordinator.

Hygiene promotion. Insufficient funding was available for study-administered hygiene promotion activities. Routine government-organized hygiene programs continued in both the intervention and control communities, primarily through the health extension worker program. ${ }^{7,8}$ Existing health extension workers with prior experience working with trachoma programs provided education about water supply and personal hygiene in community- and household-based settings.

Outcome assessments. The TANA II 42-month study visit (April 2014) is considered the baseline visit for the present trial. That visit was conducted approximately 6 months following a population census performed for the study. A random sample of 50 children aged $0-9$ years per community was drawn from the census and assessed for clinical signs of trachoma using the WHO's simplified grading system. ${ }^{9}$ Clinically active trachoma was defined as the presence of trachomatous inflammationfollicular (TF) and/or trachomatous inflammation-intense (TI), abbreviated TF $\pm \mathrm{Tl}$ in this manuscript. A conjunctival swab was then passed three times over the everted superior tarsal conjunctiva of the right eye. Swabs were placed in tubes without media and stored on ice for $<8$ hours while in the field, then at $-20^{\circ}$ for up to 4 weeks, and finally in a $-20^{\circ}$ freezer at the Amhara Public Health Institute in Bahir Dar, Ethiopia. Swabs were pooled in groups of five by community and then processed for the presence of $C$. trachomatis DNA with the Abbott RealTime assay on the $\mathrm{m} 2000$ platform. If $\geq 80 \%$ of pools from a particular community were positive, then swabs from all positive pools were processed individually. Similar methods were used for the 24-month visit of the present study (April 2016), except that assessments were performed on all 0- to 5-year-old children who had been identified at a door-to-door census conducted 24 weeks prior. This younger age-group was chosen to enhance the statistical power because ocular chlamydial infection was expected to be greater in this age-group. ${ }^{10,11}$

Process indicators. Although the local government water office was ultimately responsible for maintenance, the functionality of the water points was tracked remotely by the study coordinator through telephone calls with water officials. In addition, the study coordinator visited all water points in person at the 24-month visit to assess their functionality and ask community members about their functionality. Estimates of water availability and use and face-washing behaviors were assessed in a household survey, conducted in a random sample of approximately 10 households per community at the time of the 24month monitoring visit. Ten households were chosen because this was a feasible number for our team to complete in a single day. The household survey was administered to the head of household or spouse at their residence by a set of specifically trained workers. No direct observations of face-washing behaviors were made as part of this study (e.g., as a structured observation study might do) because of limited resources.

Statistics. The prespecified primary outcome was the community-level prevalence of ocular chlamydia in 0- to 5-yearold children at the 24-month study visit, as estimated from the pooled swabs using maximum likelihood methods. ${ }^{12}$ The community prevalence of clinically active trachoma (i.e., $\mathrm{TF} \pm \mathrm{TI}$ ) in the same age-group at month 24 was a prespecified secondary outcome. The primary analysis was a Wilcoxon rank-sum test, used to compare community-level estimates between the two arms in an 
intention-to-treat analysis. Prevalence estimates were summarized as a Lehman-Hodges pseudo-median with bootstrapped $95 \% \mathrm{Cl}$ drawn from 9,999 replications. Linear regression of the community-level prevalence estimates was performed to provide an estimate of the magnitude of the association after adjustment for baseline prevalence. Fourteen communities provided $80 \%$ power to determine a $17 \%$ difference in ocular chlamydia between the two arms, assuming an SD of 0.10 from the TANA trial and a two-sided alpha of $0.05 .{ }^{13}$ An effect size of this magnitude could be plausible if, after many years of mass azithromycin distributions, the intervention reduced transmission enough that the prevalence of chlamydia stayed low, whereas infection would be expected to return in the control communities. ${ }^{14}$

\section{RESULTS}

Fourteen communities drawn from the TANA II study were randomized to either the HDW group or to the control group (Figure 1). Characteristics were similar in the two groups before randomization (Table 1). Although children in all communities had been treated with mass antibiotic distributions at least annually for the preceding 7 years, approximately onethird of children aged 0-9 years had inflammatory signs of trachoma (i.e., TF and/or TI) before randomization for this trial. Ocular chlamydia infection was much lower, with a median of 0\% (IQR: 0-4\%) in the HDW group and 6\% (IQR: 1-6\%) in the control group.

A HDW was successfully constructed in six of the seven intervention communities. Construction was completed in each of the six communities within 4 months of randomization. In the seventh community, no water was found at two different locations, and local water officials advised against digging at a third location. Communities continued to receive government health services for the remainder of the study period, but received no programmatic mass azithromycin distributions or hygiene interventions. Of the six constructed HDWs, three were functional year-round, two were functional in the wet season only, and one ceased functioning after 3 months. Of

\section{Enrollment}

\section{Allocation}

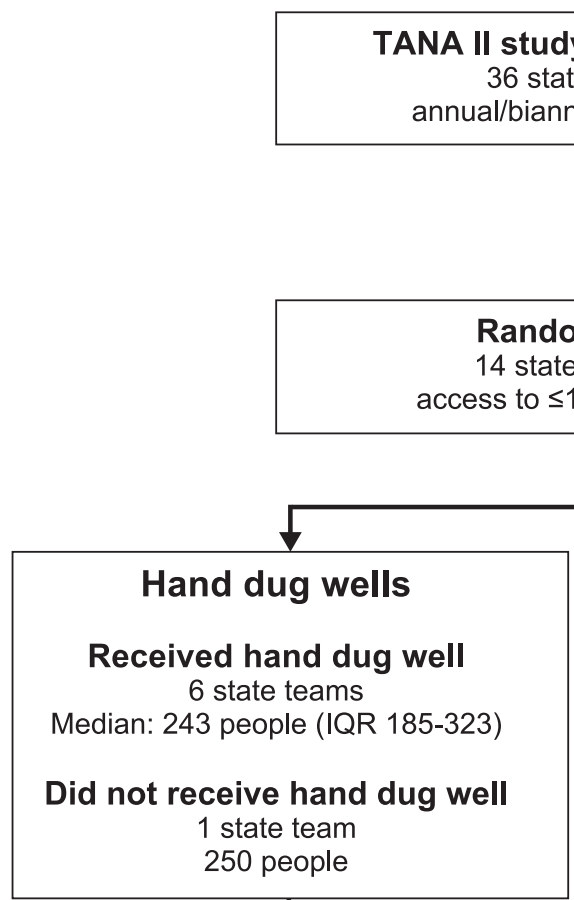

\section{Follow-up}

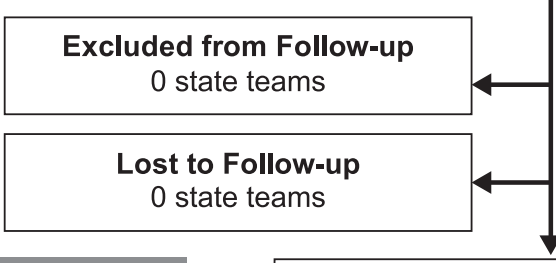

\section{Analysis}

Assessed for ocular chlamydia and included in analysis

Month 24

7 state teams

Median: 18 children 0-5y (IQR 17-25)
Excluded

0 state teams

Did not meet inclusion criteria 12 remote state teams

7 state teams with $>1$ water point 1 state team with no site for water

Refused

2 state teams

Figure 1. CONSORT flow diagram. 
TABLE 1

Baseline characteristics of communities

\begin{tabular}{lcc}
\hline & & Median (interquartile range) \\
\cline { 2 - 3 } \multicolumn{1}{c}{ Characteristic } & Hand-dug wells $(N=7)$ & Control $(N=7)$ \\
\hline Distance to nearest town $(\mathrm{km})$ & $5.4(4.8-5.6)$ & $6.6(5.3-9.4)$ \\
Altitude $(\mathrm{m})$ & $2,624(2,613-2,644)$ & $2,634(2,573-2,683)$ \\
Population, $N$ & $250(197-304)$ & $239(199-417)$ \\
Children 0-9 years, \% & $26(24-28)$ & $24(22-25)$ \\
Trachoma $(\mathrm{TF} \pm \mathrm{TI}), \% \dagger$ & $30(19-48)$ & $31(25-34)^{\star}$ \\
Chlamydia, \%† & $0(0-0)$ & $2(0-6)^{*}$ \\
\hline
\end{tabular}

$\mathrm{TF} \pm \mathrm{TI}=$ follicular trachoma and/or intense trachoma according to the WHO's simplified grading system.

$\dagger$ Assessed in a random sample of children aged $0-9$ years

the three functional water points, water flow was measured to be 10,19 , and $21 \mathrm{~L}$ per second at the final study visit. Difficulties with water production were thought to be at least in part due to the severe drought affecting Ethiopia during the study period. No harms or unintended effects were reported in either study arm during the trial.

A household survey was performed in a random sample of households from all 14 study communities at the 24-month monitoring visit, in the dry season (Table 2). This survey found self-reported travel times for water collection to be slightly lower in the intervention arm, with approximately three-quarters of households reporting traveling less than 30 minutes in the intervention arm compared with about half of households in the control arm. Ultimately, however, the survey demonstrated little difference in water consumption or face-washing behavior between communities in the two treatment arms across a variety of indicators. Most households in intervention communities continued to use non-study water points.
Community-level estimates of $\mathrm{TF} \pm \mathrm{TI}$ and ocular C. trachomatis are shown in Table 3. At month 24, chlamydia infection among 0- to 5-year-olds was not statistically significantly different between the two groups, with a pseudomedian of $23 \%$ (95\% Cl: $6-32 \%)$ in the HDW group and $13 \%$ (95\% Cl: 6-19\%) in the control group (Wilcoxon rank-sum test $P>0.99$; primary intention-to-treat analysis). The prevalence of ocular chlamydia was on average $8 \%$ higher $(95 \% \mathrm{Cl}: 5 \%$ lower to $23 \%$ higher) in the HDW communities after controlling for baseline chlamydia prevalence. The prevalence of TF $\pm \mathrm{TI}$ at month 24 was also not statistically significantly different between the two groups, with a pseudo-median prevalence of 54\% (95\% Cl: 44-64\%) in the HDW group and 43\% (IQR: $31-52 \%$ ) in the control group (Wilcoxon rank-sum test $P=$ $0.16)$. The prevalence of TF \pm TI was on average $12 \%$ higher in the HDW communities (95\% Cl: $2 \%$ lower to $27 \%$ higher) after adjusting for baseline TF \pm TI prevalence. Estimates of trachoma and ocular chlamydia were not demonstrably lower in

TABLE 2

Household survey results at 24-month study visit

\begin{tabular}{|c|c|c|}
\hline \multirow[b]{2}{*}{ Characteristic } & \multicolumn{2}{|c|}{ Mean $(95 \% \mathrm{Cl})$} \\
\hline & HDWs $(N=7)$ & Control $(N=7)$ \\
\hline Households surveyed per community & $12(11-15)$ & $10(10-11)$ \\
\hline \multicolumn{3}{|l|}{ Fraction of households } \\
\hline Using the study water point in past week, \% & $13 \%(0-32 \%)$ & $0 \%(0-0 \%)$ \\
\hline Using a protected HDW in past week, \% & $45 \%(12-77 \%)$ & $43 \%(17-71 \%)$ \\
\hline Using an unprotected HDW in past week, \% & $14 \%(0-50 \%)$ & $0 \%(0-0 \%)$ \\
\hline Using an unprotected spring well in past week, \% & $40 \%(9-73 \%)$ & $44 \%(12-77 \%)$ \\
\hline Using an open water body in past week, $\%$ & $0 \%(0-0 \%)$ & $13 \%(0-30 \%)$ \\
\hline Traveling < 30 minutes per day collecting water, past week, \% & $74 \%(59-89 \%)$ & $45 \%(21-67 \%)$ \\
\hline Reporting enough water for face washing yesterday, $\%$ & $100 \%(100-100 \%)$ & $100 \%(100-100 \%)$ \\
\hline With latrine observed, $\%$ & $65 \%(53-79 \%)$ & $53 \%(36-70 \%)$ \\
\hline With soap observed, $\%$ & $56 \%(20-90 \%)$ & $29 \%(8-50 \%)$ \\
\hline Mean distance from house to potential water point $(\mathrm{km})$ & $0.4(0.3-0.4)$ & $0.5(0.3-0.7)$ \\
\hline Mean total time spent collecting water per day (minute) & $53(38-66)$ & $60(39-80)$ \\
\hline Mean time spent traveling to collect water per day (minute) & $21(14-27)$ & $30(21-41)$ \\
\hline Mean time spent queuing for water per day (minute) & $32(17-47)$ & $30(13-47)$ \\
\hline Mean daily volume of water collected per household (L) & $67(55-78)$ & $70(62-81)$ \\
\hline Mean daily volume of water collected per capita (L) & $14(12-16)$ & $15(13-18)$ \\
\hline Mean daily volume of water used for face washing per capita $(\mathrm{L})$ & $0.7(0.6-0.9)$ & $0.7(0.5-0.8)$ \\
\hline \multicolumn{3}{|l|}{ Fraction of households in which a randomly selected } \\
\hline Child aged $0-5$ years had their face washed yesterday, \%* & $99 \%(96-100 \%)$ & $99 \%(95-100 \%)$ \\
\hline Child aged 6-9 years washed their face yesterday, \%† & $96 \%(89-100 \%)$ & $97 \%(90-100 \%)$ \\
\hline Person $\geq 10$ years washed their face yesterday, $\%$ & $99 \%(96-100 \%)$ & $100 \%(100-100 \%)$ \\
\hline Child aged $0-5$ years had their face washed with soap yesterday, \%* & $46 \%(26-65 \%)$ & $54 \%(39-66 \%)$ \\
\hline Child aged $6-9$ years washed their face with soap yesterday, \%† & $38 \%(19-56 \%)$ & $32 \%(13-51 \%)$ \\
\hline Person $\geq 10$ years washed their face with soap yesterday, $\%$ & $31 \%(14-49 \%)$ & $20 \%(11-30 \%)$ \\
\hline
\end{tabular}

HDW $=$ hand-dug well. Community-level means are shown, with bootstrapped $95 \%$ Cls $(9,999$ replications).

* Of households with a 0 - to 5 years-old child; 86 of 87 households from the HDW arm and 69 of 72 households from the control arm contributed data.

† Of households with a 6- to 9 years-old child; 40 of 87 households from the HDW arm and 28 of 72 households from the control arm contributed data. 
TABLE 3

Estimates of ocular chlamydia infection and clinically active trachoma

\begin{tabular}{|c|c|c|c|c|c|}
\hline \multirow[b]{2}{*}{ Community } & \multirow[b]{2}{*}{ Functionality } & \multicolumn{2}{|c|}{ Baseline prevalence, $0-9$ years } & \multicolumn{2}{|c|}{ Twenty four-month prevalence, $0-5$ years } \\
\hline & & $\mathrm{TF} \pm \mathrm{TI}$ & Chlamydia & $\mathrm{TF} \pm \mathrm{TI}$ & Chlamydia \\
\hline \multicolumn{6}{|c|}{ Hand-dug well } \\
\hline 1 & Year-round & $29 / 58(50 \%)$ & 0/42 (0\%) & $21 / 31(68 \%)$ & $2 / 31(6 \%)$ \\
\hline 2 & Year-round & $26 / 56(46 \%)$ & $0 / 56(0 \%)$ & $24 / 37(65 \%)$ & $12 / 37(32 \%)$ \\
\hline 3 & Year-round & $10 / 50(20 \%)$ & $0 / 50(0 \%)$ & 8/16 (50\%) & $0 / 16(0 \%)$ \\
\hline 4 & Wet season & $11 / 37$ (30\%) & $0 / 37(0 \%)$ & $8 / 17(47 \%)$ & $0 / 17(0 \%)$ \\
\hline 5 & Wet season & 7/37 (19\%) & $0 / 37(0 \%)$ & $8 / 16(50 \%)$ & $0 / 16(0 \%)$ \\
\hline 6 & First 3 mos & $6 / 36(17 \%)$ & $0 / 36(0 \%)$ & $7 / 18(39 \%)$ & $0 / 18(0 \%)$ \\
\hline 7 & Never & $32 / 50(64 \%)$ & $2 / 50(4 \%)$ & $11 / 18(61 \%)$ & $5 / 19(26 \%)$ \\
\hline \multicolumn{6}{|l|}{ Control } \\
\hline 8 & - & $23 / 53(43 \%)$ & $2 / 53(4 \%)$ & $2 / 10(20 \%)$ & $0 / 10(0 \%)$ \\
\hline 9 & - & $9 / 26(35 \%)$ & $0 / 28(0 \%)$ & $4 / 12(0 \%)$ & $0 / 12(0 \%)$ \\
\hline 10 & - & $15 / 45(33 \%)$ & $4 / 45(9 \%)$ & $20 / 39(51 \%)$ & $7 / 39(18 \%)$ \\
\hline 11 & - & $14 / 49(29 \%)^{\star}$ & $6 / 50(12 \%)^{\star}$ & $19 / 36(53 \%)$ & 7/36 (19\%) \\
\hline 12 & _- & $15 / 53(28 \%)$ & $4 / 53(8 \%)$ & $18 / 32(56 \%)$ & $2 / 32(6 \%)$ \\
\hline 13 & - & $8 / 34(24 \%)$ & $0 / 34(0 \%)$ & $6 / 14(43 \%)$ & $0 / 14(0 \%)$ \\
\hline 14 & _- & $6 / 42(14 \%)$ & $0 / 42(0 \%)$ & $5 / 13(39 \%)$ & $1 / 13(8 \%)$ \\
\hline
\end{tabular}
*Missing data at time point. Values reflect the most recent study visit in the TANA II trial (i.e., last observation carried forward imputation); no mass azithromycin distribution was administered in the intervening time.

the three communities with a functional water point for the entire year (Table 3).

\section{DISCUSSION}

We were unable to demonstrate that construction of HDWs, performed as a sole intervention without other hygiene promotion activities, prevented transmission of ocular chlamydia in this small cluster-randomized trial. We found no evidence to suggest that water point construction alone resulted in any behavioral changes with regard to face-washing, although this was assessed only by self-report in a random sample of households at the conclusion of the trial. Communities in which a water point provided water throughout the year did not appear to fare better than communities with less reliable HDWs. The use of constructed HDWs was generally low, even in communities with more reliable HDWs.

The WHO's SAFE strategy (i.e., surgery, antibiotics, facial cleanliness, and environmental improvements) promotes environmental improvements such as increased access to water to reduce trachoma transmission. Ocular secretions are thought to be the chief reservoir of chlamydia for a community, so facewashing should theoretically remove these secretions and reduce the source of reinfection. This theory is supported by numerous observational studies that have found an association between facial cleanliness and ocular chlamydial infection. ${ }^{15-18}$

The results of the present study are consistent with previous randomized trials, none of which have provided strong evidence supporting the efficacy of water point construction for trachoma. One trial in Niger randomized six communities to a community HDW plus education and mass azithromycin, and another six communities to mass azithromycin alone. ${ }^{5}$ No difference in ocular chlamydia was observed between the two groups of communities at the 2-year time point, although little information about treatment fidelity was provided (e.g., when water point construction was completed, whether the water points were functional, whether water was being used by households, and whether face-washing increased). Other trials have provided face-washing education but no hardware to increase the volume of available water. ${ }^{19}$ The most promising results came from two small cluster-randomized trials. ${ }^{20,21}$ One of these trials enrolled three pairs of villages, randomizing each village of the pair to either face-washing education or no intervention, and found a lower prevalence of the secondary outcome of $\mathrm{TI}$ in two of the pairs, but not in the third. ${ }^{20}$ Another trial randomized four villages to topical tetracycline, health education, tetracycline plus health education, or no intervention, and found a lower incidence of TF \pm $\mathrm{TI}$ in the health education villages than in the control village. ${ }^{21}$ However, neither of these trials accounted for the clusterrandomized design, and their small sample size limits statistical conclusions and generalizability. Other randomized trials have not suggested a positive effect of hygiene education on trachoma. $^{22-24}$

We hypothesized that constructing a community water point would increase the volume of water available for facewashing. However, households in the HDW arm did not report collecting or using a greater volume of water than those in the intervention communities at the 24-month household survey. Several limitations of the study could explain this finding. First, the HDWs did not universally provide more water for the intervention communities. No water was found at one of the sites, the water dried up after 3 months at another, and water was available only during the wet season in two others. Although the household survey suggested slightly lower travel times for the intervention arm, the volume of water collected was ultimately similar in the two arms. Protected water sources were present in all communities before the trial, and the household survey revealed that many families continued to use non-study water points-even in communities that had a functional study water point. Second, the sample size of the trial was small, increasing the likelihood of imbalanced randomization and chance accounting for the findings. Third, water is a valuable resource with many uses. Even if the volume of available water were increased, households may have decided to use the extra water for activities other than facewashing. Moreover, due to a lack of resources, our intervention did not include any hygiene education component or household-level hardware that might promote personal hygiene (e.g., tippy taps and soap). It is possible that improving 
access to water will not have health effects unless accompanied by hygiene promotion programs. ${ }^{25}$ In the case of the present study, community norms for washing may have been set by the realities and challenges of water collection, because the vast majority of households thought they had sufficient water for washing, and the vast majority of respondents reported washing their face yesterday, despite the lack of water relative to high-income settings. Thus, an intervention with health messaging specifically targeting personal hygiene may increase the amount of water used for personal hygiene. Finally, water use is a complicated practice, and people do not routinely measure the exact quantities of water used for various activities, making it challenging to estimate the volume of water used for cooking, cleaning, and drinking. We assessed water use based on self-reported questionnaire, subjecting these estimates to misclassification. The generalizability of the results to other areas is not certain, especially to areas with a different baseline level of water availability or different endemicity of trachoma.

This trial highlights that simply constructing a HDW does not guarantee its functionality, and even a functional water point does not automatically improve face-washing behavior or increase the volume of water used for hygiene-all of which would be important mediators in the causal pathway explaining an association between water points and trachoma. Thus, this study cannot confirm whether facial cleanliness itself has a causal relationship with trachoma, but rather can only conclude that in this particular study area, installation of a water point, in the absence of any other dedicated hygiene interventions, was not associated with reductions in ocular chlamydia. It is certainly possible that water points are an important intervention for trachoma control, but require concomitant hygiene promotion activities to be effective.

In conclusion, this small cluster-randomized trial set in an area with hyperendemic trachoma did not find an association between community HDW construction and reduced ocular chlamydia; however, it is important to note that the intervention communities did not receive any additional hygiene promotion activities aside from the water point installation. The difficulties we encountered in finding water and maintaining the water points through local committees confirmed the practical difficulties that trachoma programs face when trying to increase community water supply. Despite the financial burden and logistical challenges associated with water, sanitation, and hygiene (WASH) interventions, we believe that randomized trials are of paramount importance to better assess the causal relationship between WASH infrastructure and trachoma, and will be helpful in guiding trachoma programs toward their ultimate goal of eliminating trachoma as a public health problem.

Received April 30, 2020. Accepted for publication December 18, 2020. Published online February 1, 2021.

Note: Supplemental materials appear at www.ajtmh.org.

Acknowledgments: We thank the Goncha Siso Enese woreda health office and the health extension workers who helped coordinate the study, as well as Abbott for its donation of the m2000 RealTime molecular diagnostic system and consumables.

Financial support: The main funders of the trial were That Man May See and the National Eye Institute (grant U10 EY016214). Additional funding was provided by the Bernard Osher Foundation, the Harper Inglis Trust, the Bodri Foundation, the Proctor Foundation's South
Asia Research Fund, and Research to Prevent Blindness. The trachoma control program in Amhara is supported by the Carter Center, Amhara Regional Health Bureau, the Lions-Carter Center SightFirst Initiative, and many individual donors.

Authors' addresses: Solomon Aragie, Sintayehu Gebresillasie, Ambahun Chernet, Ayalew Shiferaw, Zerihun Tadesse, and Mulat Zerihun, The Carter Center Ethiopia, Addis Ababa, Ethiopia, E-mails: solomon.aragie@cartercenter.org, sintayehugs@gmail.com, ambahun. chernet@cartercenter.org, ayalew.shiferaw@cartercenter.org, zerihun. tadesse@cartercenter.org, and mulat.zerihun@cartercenter.org. Nicole E. Varnado, Sun Y. Cotter, Dionna M. Wittberg, and Zhaoxia Zhou, Kristen Aiemjoy, and Jeremy D. Keenan, Francis I Proctor Foundation, University of California, San Francisco, San Francisco, CA, E-mails: nicole.e.varnado@kp.org, sun.cotter@ucsf.edu, dionna.wittberg@ ucsf.edu, zhaoxia.zhou@ucsf.edu, kaiemjoy@stanford.edu and jeremy.keenan@ucsf.edu. Elizabeth Kelly Callahan and Scott D. Nash, The Carter Center, Atlanta, GA, E-mails: kelly.callahan@ cartercenter.org and scott.nash@cartercenter.org.

\section{REFERENCES}

1. West S, Munoz B, Bobo L, Quinn TC, Mkocha H, Lynch M, Mmbaga BB, Viscidi R, 1993. Nonocular Chlamydia infection and risk of ocular reinfection after mass treatment in a trachoma hyperendemic area. Invest Ophthalmol Vis Sci 34: 3194-3198.

2. Stocks ME, Ogden S, Haddad D, Addiss DG, McGuire C, Freeman $M C, 2014$. Effect of water, sanitation, and hygiene on the prevention of trachoma: a systematic review and meta-analysis. PLoS Med 11: e1001605.

3. Solomon AW et al., 2003. Strategies for control of trachoma: observational study with quantitative PCR. Lancet 362: 198-204.

4. House $\mathrm{Jl}$ et al., 2009. Assessment of herd protection against trachoma due to repeated mass antibiotic distributions: a cluster-randomised trial. Lancet 373: 1111-1118.

5. Abdou A, Munoz BE, Nassirou B, Kadri B, Moussa F, Baare I, Riverson J, Opong E, West SK, 2010. How much is not enough? A community randomized trial of a Water and Health Education programme for Trachoma and Ocular $C$. trachomatis infection in Niger. Trop Med Int Health 15: 98-104.

6. Gebre T et al., 2012. Comparison of annual versus twice-yearly mass azithromycin treatment for hyperendemic trachoma in Ethiopia: a cluster-randomised trial. Lancet 379: 143-151.

7. Teklehaimanot HD, Teklehaimanot A, 2013. Human resource development for a community-based health extension program: a case study from Ethiopia. Hum Resour Health 11: 39.

8. Banteyerga $\mathrm{H}, 2011$. Ethiopia's health extension program: improving health through community involvement. MEDICC Rev 13: 46-49.

9. Thylefors B, Dawson CR, Jones BR, West SK, Taylor HR, 1987. A simple system for the assessment of trachoma and its complications. Bull World Health Organ 65: 477-483.

10. Solomon AW et al., 2004. Mass treatment with single-dose azithromycin for trachoma. N Engl J Med 351: 1962-1971.

11. West ES, Munoz B, Mkocha H, Holland MJ, Aguirre A, Solomon AW, Bailey R, Foster A, Mabey D, West SK, 2005. Mass treatment and the effect on the load of Chlamydia trachomatis infection in a trachoma-hyperendemic community. Invest Ophthalmol Vis Sci 46: 83-87.

12. Diamant J, Benis R, Schachter J, Moncada J, Pang F, Jha HC, Bhatta RC, Porco T, Lietman T, 2001. Pooling of Chlamydia laboratory tests to determine the prevalence of ocular Chlamydia trachomatis infection. Ophthalmic Epidemiol 8: 109-117.

13. Stoller NE et al., 2011. Efficacy of latrine promotion on emergence of infection with ocular Chlamydia trachomatis after mass antibiotic treatment: a cluster-randomized trial. Int Health 3: 75-84.

14. Lakew T et al., 2009. Reduction and return of infectious trachoma in severely affected communities in Ethiopia. PLoS Negl Trop Dis 3: e376.

15. Burton MJ et al., 2003. Which members of a community need antibiotics to control trachoma? Conjunctival Chlamydia trachomatis infection load in Gambian villages. Invest Ophthalmol Vis Sci 44: 4215-4222. 
16. Harding-Esch EM et al., 2010. Trachoma prevalence and associated risk factors in the Gambia and Tanzania: baseline results of a cluster randomised controlled trial. PLoS Negl Trop Dis 4: e861.

17. Ayele B et al., 2011. Risk factors for ocular chlamydia after three mass azithromycin distributions. PLoS Negl Trop Dis 5: e1441.

18. Amza A et al., 2012. Community risk factors for ocular Chlamydia infection in Niger: pre-treatment results from a clusterrandomized trachoma trial. PLoS Negl Trop Dis 6: e1586.

19. Ejere HO, Alhassan MB, Rabiu M, 2015. Face washing promotion for preventing active trachoma. Cochrane Database Syst Rev 4: CD003659.

20. West S, Munoz B, Lynch M, Kayongoya A, Chilangwa Z, Mmbaga BB, Taylor HR, 1995. Impact of face-washing on trachoma in Kongwa, Tanzania. Lancet 345: 155-158.

21. Resnikoff S, Peyramaure F, Bagayogo CO, Huguet P, 1995. Health education and antibiotic therapy in trachoma control. Rev Int Trach Pathol Ocul Trop Subtrop Sante Publique 72: $101-110$
22. Peach $H$, Piper $S$, Devanesen $D$, Dixon $B$, Jeffries $C$, Braun $P$, Nelson D, Kruger G, Boulden H, 1987. Northern Territory Trachoma Control and Eye Health Committee's Randomised Controlled Trial of the Effect of Eye Drops and Eye Washing on Follicular Trachoma Among Aboriginal Children. Darwin, Australia: Menzies School of Health Research.

23. Edwards T, Cumberland P, Hailu G, Todd J, 2006. Impact of health education on active trachoma in hyperendemic rural communities in Ethiopia. Ophthalmology 113: 548-555.

24. Atik B, Thanh TT, Luong VQ, Lagree S, Dean D, 2006. Impact of annual targeted treatment on infectious trachoma and susceptibility to reinfection. JAMA 296: 1488-1497.

25. Hulland K, Martin N, Dreibelbis R, 2015. What Factors Affect Sustained Adoption of Safe Water, Hygiene and Sanitation Technologies? A Systematic Review of Literature. Available at: https://eppi.ioe.ac.uk/cms/Portals/0/PDF\%20reviews\%20and\% 20summaries/WASH\%20technologies\%202015\%20Hulland\% 20report.pdf?ver=2015-06-10-141853-910. Accessed November 13,2020 . 